KfK 4252

Juli 1987

\title{
Interaction of Local Instabilities during Oscillatory Convection
}

J. N. Koster Institut für Reaktorbauelemente

Kernforschungszentrum Karlsruhe 
KERNFORSCHUNGSZENTRUM KARLSRUHE

Institut für Reaktorbauelemente

KfK 4252

INTERACTION OF LOCAL INSTABILITIES DURING

OSCILLATORY CONVECTION

Jean N. Koster*

* present address: University of Colorado, Department of Aerospace Engineering Sciences, Boulder, Colorado 80309-0429

Kernforschungszentrum Karlsruhe GmbH, Kar1sruhe 
Als Manuskript vervielfältigt

Für diesen Bericht behalten wir uns alle Rechte vor

Kernforschungszentrum Karlsruhe $\mathrm{GmbH}$

Postfach 3640, 7500 Karlsruhe 1

ISSN 0303-4003 


\section{Abstract}

This paper studies the time dependent convective flows in Hele-Shaw slots which are heated from below. In this experimental study, the evolution of oscillatory phenomena is traced through the time dependent regime which is bounded on either side by regions of steady flow. The history of thermal events is recorded by thermocouples which protrude into the test fluid (silicone oil, $\operatorname{Pr} \cong 37$ ) at the four corners of the cavity. Flow patterns are visualized by holographic interferometry and temperature signals are analyzed using Fourier transforms and correlation functions.

The results of this investigation show that the richness of the dynamic behavior of time dependent convective flows in high Prandt 1 number fluids is determined by the interaction of individual, spatially distributed instability events. Additionally, this behavior is dependent on the symmetry of the flow pattern which governs phase-locking properties of thermal plumes developing at different locations. 
Wechselwirkung lokaler Instabilitäten bei oszillatorischer Konvektion Zusammenfassung:

Dieser Bericht untersucht zeitabhängige Konvektionsströmungen in von unten beheizten Hele-Shaw-Spalten. In dieser experimentellen Arbeit wird die Entwicklung oszillatorischer Phänomene verfolgt. Sie findet in einem zeitabhängigen Bereich statt, der beidseitig von stationären Strömungszuständen begrenzt wird. Das thermische Verhalten wird mit Thermoelementen registriert, die in den vier Ecken der Zelle in die Experimentierfliissigkeit (Silikonöl, $\mathrm{Pr}=37)$ ragen. Die Strömungsmuster werden mittels holographischer Interferometrie sichtbar gemacht; die Temperatursignale werden mit Hilfe von FourierTransformationen und Korrelationsfunktionen analysiert.

Die Ergebnisse dieser Untersuchung zeigen, daß die Vielfältigkeit des dynamischen Verhaltens zeitabhängiger Strömungen in Flüssigkeiten mit hohen Prandtzahlen von den Wechselwirkungen einzelner, räumlich verteilter Instabilitäten bestimmt wird. Die Symmetrie des Strömungsmusters ist dabei verantwortlich für die Phasenkopplung von thermischen "Plumes", die an verschiedenen Orten entstehen. 
1. Introduction 1

1.1 Background 1

1.2 Thermal Boundary Layers 3

1.3 Aims 4

2. Experimenta1

3. Experimental Results 9

3.1 Flow Patterns 9

3.2 Frequency Analysis 12

3.3 Correlation Analysis 22

4. Discussion $r$

5. Summary and Conclusion 13

6. References $\quad 32$ 


\section{Introduction}

1.1 Background

While investigating the Malkus (1954a, b) heat transfer transitions in large fluid layers, Somerscales \& Dropkin (1966) and Willis \& Deardorff (1965, 1970) observed a transition regime of periodic flow between steady and nonperiodic thermal convection. Mitchell \& Quinn (1966), in examining this region between steady and nonperiodic thermal convection, demonstrated that in small cylindrical convection chambers, complex time-periodic signals develop after passing a regime of monoperiodic oscillations. However, Mitchell \& Quinn did not perform a detailed statistical analysis of these signals. More exhaustive investigations were to arrive later in the studies of Ahlers (1974), Ahlers \& Behringer (1978a, b), Berge \& Dubois (1976), Gollub \& Benson (1978, 1980), Libchaber \& Maurer (1978, 1980) and others as the work focused on frequency analysis of signals monitored in time dependent convective flows. These more rigorous investigations uncovered an array of phenomena, such as periodicity, quasiperiodicity, frequency locking, intermittency, subharmonics and nonperiodicity. Most of that work, as does the present study, concentrates on small containers.

The transition to time dependent flow also received more theoretical attention. The early models developed by Hopf (1948) and Landau \& Lifshitz (1959) described the transition to turbulence as an infinite set of successive bifurcations. After several bifurcations the behavior of the solutions becomes continuously more complicated which leads to their picture of the transition to turbulence. This model has some bearings on convection in extended layers but seems not to apply to convection in small boxes.

An alternative theory has been presented by Ruelle \& Takens (1971) and Newhouse, Ruelle \& Takens (1978). They suggest that when increasing the stress parameter in their model equations, the time dependent flow remains periodic until the third bifurcation. Each bifurcation is marked by the appearance of one new incommensurate frequency. After the third bifurcation, a so-called "strange attractor" is likely to bound the system in the phase space. This strange attractor implies that the time dependent solutions now show nonperiodic features. In terms of phase space trajectories such a nonperiodic flow oscillation is often referred to as turbulent or chaotic. Most of the experimental results on time dependent convection in small containers show 
some consistency with the theory of Newhouse, Ruelle \& Takens (1978) in that, generally, no more than two incommensurate frequencies can be found directly from spectra (see e.g. Gollub \& Benson, 1980; Ahlers \& Behringer, 1978a; Berge \& Dubois, 1976; Libchaber \& Maurer, 1980).

However, in some cases, experimentalists observed also a three-frequency quasiperiodicity (Gollub \& Benson, 1980). Such results were also found by Koster \& Müller (1984, hereafter referred to as I) who, in addition, observed a difference in phase of two locked frequencies. That result was considered an indication that four frequencies develop at four different locations. Walden, et al. (1984) recently found that even four and five frequency quasiperiodic oscillations may appear in a six and eight convection cell configuration. They also find that the oscillators are localized in different parts of the container. These experimental results got support from Grebogi, et al. (1983) who showed that, in complement to the findings of Newhouse, Ruelle \& Takens (1978), three frequency quasiperiodic orbits can be expected in nonlinear systems, without generating nonperiodicity. According to Grebogi et al., perturbations must have a defined quality to cause the three-frequency quasiperiodicity to cease and turn into a chatic oscillation. In other words, the statistical behavior of a many oscillator system is a function of the numerical value of a nonlinearity parameter between oscillators. In the same vein are the results presented by Koster, et al. (1986). They have shown that, in the fluid layer of a finite Hele-Shaw cell, there may exist three fluid regions within a certain range of Rayleigh numbers: the center region having a steady flow and the two end regions exhibiting time dependent flow. In the time dependent regime the two individual oscillations were always monoperiodic and uncorrelated at any Rayleigh number. A transition to a more complicated oscillation was not observed, and the flow never adopted nonperiodic behavior. That result confirms Grebogi's, et al. (1985) results that, in order to develop a transition to turbulence, a certain amount of nonlinear interaction of several flow oscillators is needed.

The wide array of observations made in the study of transitions in oscillatory convective flows, leads to the following major question: Can a linkage be established between the cause of oscillatory flows and the nonlinear behavior that is observed. The comparison of experimental data with theoretical mode1s is limited (Curry, et a1., 1984). The approach taken in the present investigation on high Prandtl number flows, is to focus attention on the 
individual thermal bundary layer areas as a possible location where instabilities may develop.

\subsection{Thermal Boundary Layers}

Based on observations that in convective flow thermal boundary layers develop at the horizontal boundaries of the fluid layer, Howard (1964) introduced a model to explain the cause of time dependent convective flow in high Prandt number fluids. He postulated that a horizontal thermal boundary layer of thickness $d$ becomes unstable when the temperature gradient across this layer reaches the critical value for the onset of convection. At this onset of convection, secondary vortices appear in the thermal boundary layers. These vortices grow and break of $f$ the thermal boundary layers in the form of thermal plumes. This time dependent release of plumes from the thermal boundary layers generates time dependent convective flow. In the case of a large horizontal layer, Sparrow, Husar \& Goldstein (1970) have visualized the break-off process of thermal plumes. Busse \& Whitehead (1974) confirmed the Howard model for high Prandt number free convection in a wide fluid layer. Berge \& Dubois (1979) confirmed the model for small boxes. Using interferometry, Koster \& Müller (1982) have further established the existence of time dependent flows, generated by secondary vortices which develop in thermal boundary layers of narrow Hele-Shaw cells.

The discussion about the applicability of the Howard model of the instability of horizontal thermal boundary layers to the special Hele-Shaw slot as a driving mechanism of flow oscillations has been introduced in I. In that study, the flow had a cellular pattern close to the horizontal boundaries: a stagnation flow along the centerline with one cell to each side. The flow pattern thus had four horizontal thermal boundary layers at the horizontal boundaries. It was apparent from that study that individual horizontal thermal boundary layers may become unstable whereas other neighbouring ones may not. Koster \& Muller (I) proposed a working hypothesis according to which frequency-locked oscillations develop from phase-locked thermal instability events in several horizontal thermal boundary layers. Their interpretation was drawn from signals monitored at midheight of the slot. Finally, they observed a strong dependence of the transitions in the oscillation character on the evolution of the flow pattern with temperature difference. 
Then, Koster (1985) evaluated the thickness $\delta$ of thermal boundary layers from interferograms. It could be shown that: (i) the thermal boundary layer thickness $\delta$ can be smaller at the lower boundaries than at the upper boundaries, (ii) the thickness $\delta$ is time dependent, ( $i i i$ ) the frequency of growth of $\delta$ is the same as the frequency monitored at the transducers located just above the thermal boundary layers.

\subsection{Aims}

The following new experiment relates to high Prandtl number oscillatory convection in a low conductivity Hele-Shaw slot. The goal of the experiment is to provide more precise knowledge aimed at uncovering the fluid dynamic mechanism that is capable of driving the oscillations in convective flow and which is capable of developing complicated signals with more than one frequency. To accomplish this goal, simultaneous measurements were made of thermal fluctuations close to the four thermal boundary layers developing at the horizontal walls. The temperature pattern of the fluid layer was visualized simultaneously by interferometry. The investigation aims at providing ân answer to the following questions: (i) Is the time dependent flow generated by unstable horizontal thermal boundary layers? (ii) How many instability events drive the observed oscillations? (iii) Do these instability events behave individually or are they coupled at any time? (iv) Do they generate equal frequencies or different ones? ( $v$ ) Are periodic (frequency-locked, subharmonic) oscillations caused by a phase-locked development of different, locally distributed instability events?

This paper is organized as follows. In section 2 the apparatus and the experimental procedure are described. The experimental results are presented in section 3. A discussion is given in section 4 and a conclusion in section 5 . 


\section{Experimental}

The experiments are performed in a low conductivity Hele-Shaw slot type cavity simflar to that described in I. A schematic of this cavity is given in figure 1 .

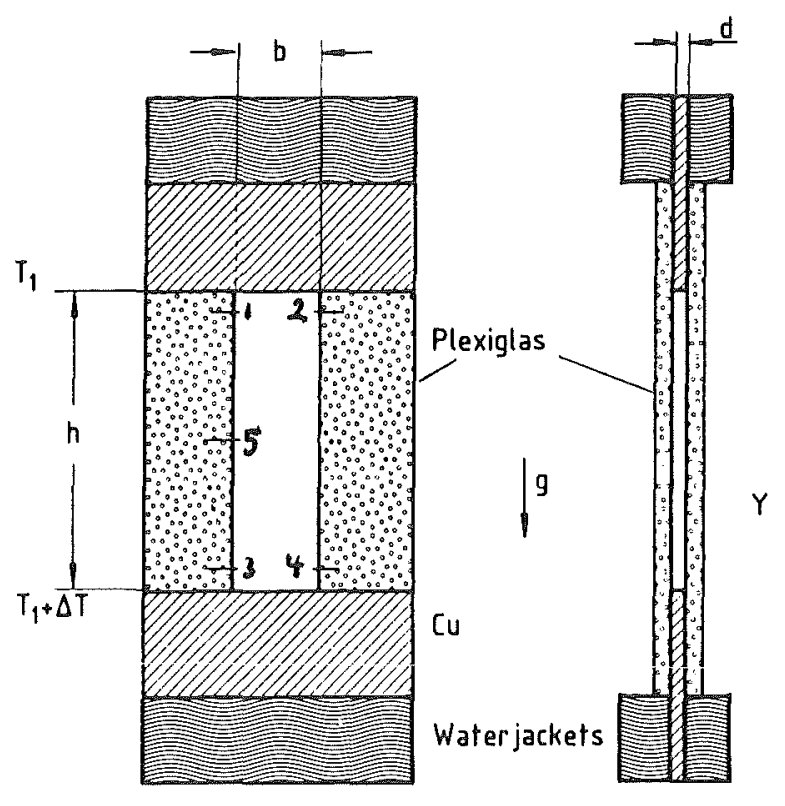

Fig. 1 - Hele-Shaw S1ot $\mathrm{h} / \mathrm{b}=3.6$. The low-conductivity slot has Plexiglas side walls. $\Delta T$ between the horizontal upper walls is measured by thermocouples across the fluid-filled gap and $\mathrm{T}_{1}$ measures the upper temperature. Numbers are assigned to the transducers that monitor time-dependent flow.

Al1 four vertical walls are made of Plexiglas. The Plexiglas windows are $2 \mathrm{~mm}$ thick, which is much less than in $\mathrm{I}$. The aspect ratio of the $\mathrm{s} l$ ot is $\mathrm{h} / \mathrm{b} / \mathrm{d}=$ $3.6 / 1 / 0.16$ with $\mathrm{d}=3.1 \mathrm{~mm}$ and an optical pathlength through the slot of $\mathrm{Y}=$ $7.25(\mathrm{Y} / \mathrm{d}=2.3)$. The gap is filled with silicone oil of viscosity $\checkmark=3$ cSt and Prandt1 number $\operatorname{Pr} \cong 37$.

The slot is fixed in a low conductivity frame (Novotex) with a second glass window and $30 \mathrm{~mm}$ thick removable insulation material in order to reduce heat coupling with the surroundings. 
The fluid layer is heated at the lower and cooled at the upper side at a symmetric heating rate of $0.02 \mathrm{~K} / \mathrm{min}$ between the measuring points taken at constant temperature difference $\Delta \mathrm{T}$ and the upper temperature $\mathrm{T}_{1}$, are measured with thermocouples in order to calculate the Rayleigh number. The physical data ( $\mathrm{I}$, table 1 ) are taken at $\mathrm{T}=\mathrm{T}_{1}+\frac{\Delta \mathrm{T}}{2}$.

The Rayleigh number is defined in the following equation:

$$
\mathrm{Ra}=\frac{\mathrm{gB} \Delta \mathrm{Th}^{3}}{\mathrm{x}}
$$

where $g$ is the acceleration of gravity, $B$ is the thermal expansion coef $f$ icient, $x$ is the thermal diffusivity, $v$ the kinematic viscosity, $\Delta T$ the temperature difference between bottom and top of the layer and $h$ is the height of the slot in the direction of gravity.

The Prandt 1 number is

$$
\operatorname{Pr}=\frac{v}{x}
$$

and the aspect ratios are $\mathrm{h} / \mathrm{d}$ and $\mathrm{h} / \mathrm{b}$ with $\mathrm{h}$, $\mathrm{d}$ and $\mathrm{b}$ defined in figure 1 . As emphasis is on the oscillatory convective flow, a normalized Rayleigh number

$$
\mathrm{Ra} *=\frac{\mathrm{Ra}}{\mathrm{Ra}_{\text {osc }}}
$$

is defined, where $\mathrm{Ra}$ osc is the critical Rayleigh number of the onset of the oscillatory convective flow defined through increasing Rayleigh number.

The time dependent flow is measured at five locations shown in figure 1. The reference temperature of a11 the $0.25 \mathrm{~mm}$ diameter chromel-Alumel thermocouples is $\mathrm{T}_{\mathrm{R}}=0 \pm 0.01{ }^{\circ} \mathrm{C}$. All thermocouples protrude $2 \mathrm{~mm}$ into the fluid. The lower and upper thermocouples are mounted $5 \mathrm{~mm}$ away from their respective horizontal walls. The time dependent signals from these five locations are amplified, their dc-offset removed, low pass filtered at the frequency of $\mathrm{f}=$ $10 \mathrm{~Hz}$ and recorded on parallel tracks of magnetic tapes. From these tape recordings, segments of $\mathrm{N}=4096$ samples are digitized with time increments of $0.5<t / s<2$. Details of further data preparation and the fast Fourier analysis are the same as given in $I$. 
Four transducers monitor the time history of the four horizontal thermal boundary layers. If one transducer monitors a time dependent signal, the adjacent thermal boundary layer is considered unstable (Koster, 1985); if a transducer monitors white noise (power $\mathrm{P}=10^{-5} \mathrm{~K}^{2} / \mathrm{Hz}$ ) the adjacent thermal boundary layer is considered stable. This measurement, however, appeared not sufficient to determine the physical cause of the instability due to a lack of visualization resolution of thermal boundary layers.

In order to measure the linear correlation of two thermocouple signals, the estimate for the cross-correlation function (Bendat \& Piersol, 1971) of the finite functions of, say $x(t)$ and $y\left(t+t^{*}\right.$ ) (when $x(t)$ and $y(t)$ are temperature signals, $t^{*}$ the time shift of the two temperature functions) may be obtained by taking the average product of the two values over the experimental observation time $t_{T^{*}}$. This estimated cross-correlation function (CCF) of the zero mean value functions $x(t)$ and $y(t)$,

$$
R_{x y}\left(t^{m}\right)=\frac{1}{t_{T}} \int_{0}^{T} x(t) y\left(t+t^{\prime \prime}\right) d t
$$

is calculated through fast Fourier transform procedures. After scaling, the correlation coefficients $\mathrm{C}_{\mathrm{xy}}(t)$ are calculated as

$$
C_{x y}(t)=\frac{R_{x y}(t)}{\sqrt{\left[R_{x x}(0) R_{y y}(0)\right]}}
$$

where $R_{x x}(0)$ and $R_{y y}(0)$ are the values of the auto-correlation function estimate of $x(t)$ and $y(t)$, respectively, for zero shift. The auto-correlation function (ACF) is calculated by setting $x(t)=y(t)$.

With the ACF it is possible to detect a periodic signal in an apparently noisy function. An ACF can also detect signal delay times of events causing the time dependence. With the CCF we will analyze the degree of correlation at different delay times of two transducer signals obtained at different locations. The result is a measure for the intensity of the coupling of oscillatory events which are visualized plumes coming from thermal boundary layer areas.

Laminar Hele-Shaw flow is considered a two-dimensional flow (Bear, 1972) and is thus ideally suited for flow visualization. The flow patterns are visualized by holographic real time interferometry. The Plexiglas windows are only 
$2 \mathrm{~mm}$ thick in order to reduce the numer of Plexiglas borne fringes in the interferograms (Koster, 1983). The other Plexiglas side walls permit a qualitative visualization of the temperature field at the smaller sides of the slot.

The accuracy of the measurements given in I applies also to this experiment. It has to be kept in mind that here we adopt a relative accuracy of $\mathrm{Ra}^{*}$ based on the accuracy of the vertical temperature difference $\Delta^{T}$ only, which is about $\pm 0.01 \mathrm{~K}$. Additional noise sources are the reference temperature device and the high dc-offset which is needed to adjust the time dependent signals to the recorder. The reference temperature device and the electronics drifted very slowly, far below the fluid flow oscillation frequencies and are thus negligible. Following Gray \& Giorgini (1976) the validity of the Boussinesq approximation, based on constant material properties, ends at $\mathrm{Ra}^{*}=4.0$. 


\section{Experimental results}

The experiments were conducted as up-scan experiments, i.e., with increasing temperature difference starting at $\Delta T=0 \mathrm{~K}$. Thus, the critical Rayleigh number for the onset of oscillations $\mathrm{Ra}_{\text {osc }}=2.79 \times 10^{7}\left(\mathrm{~T}_{\text {osc }}=1.85 \mathrm{~K}\right)$ was defined through increasing temperature difference. This threshold is the same as in $I$.

Subsequently the flow pattern analysis will be discussed. In section 3.2 the results of the frequency analysis of temperature signals are presented and in section 3.3 specific auto- and cross-correlation results are discussed.

\subsection{Flow Patterns}

Two main experiments were performed during the course of the present study. Each run was started with an isothermal convection chamber and the Plexiglas walls saturated with silicone oil. During the one week lasting first quasisteady approach of the onset of oscillations, the interferograms filled up with spurious fringes and the quality of the interferograms deteriorated with time. In order to get high quality interferograms of the flow pattern, a separate, five day lasting experiment was performed with a slightly transient one day approach ( $T$ was increased at a rate of $0.02 \mathrm{k} / \mathrm{min}$ ) of an oscillatory flow at $\mathrm{Ra}^{*}=1.73$ starting from a new hologram taken at $\Delta \mathrm{T}=0 \mathrm{~K}$. Interferograms of that oscillatory flow are shown in figure $2 a$. The flow pattern was very similar to the pattern around that Rayleigh number in the experiment described in section 3.2 , which shows that the experiment is reproducible.

The improved flow visualization of convection in this low conductivity HeleShaw slot revealed that the oscillatory flow pattern is a nonsymmetric twosided oscillation (figure 2a, b). Close to the horizontal boundaries a cellular type flow exists with stagnation points close to the vertical centerline (c. f. I). The fluid exchange zone is roughly between $h / 4$ and $h / 2$ away from the lower boundary. The flow in the center of the slot consists of a zone where the heat transport is dominated by rising plumes and their sinking counterparts. The plumes break off the vertical walls and shift horizontally either by the value $b / 2$ or $b$. Basically the flow pattern is asymetric to horizontal and vertical centerlines. Similar findings were reported in $\mathrm{I}$. 


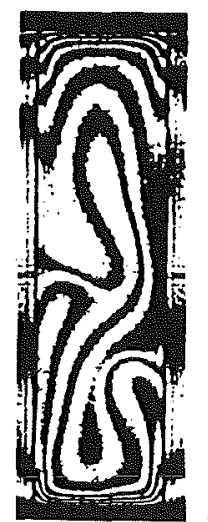

1.

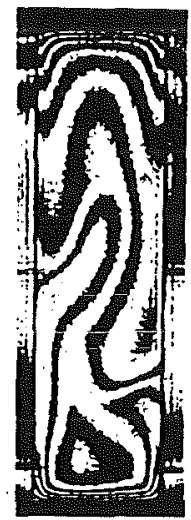

7

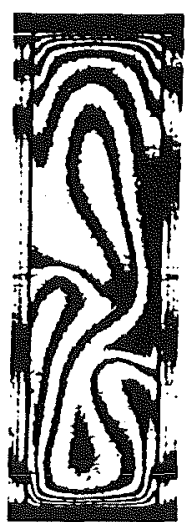

2

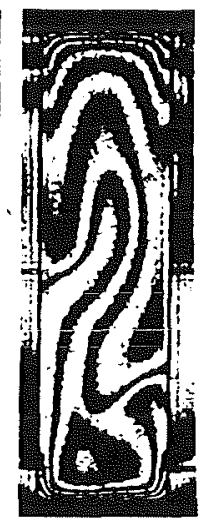

8

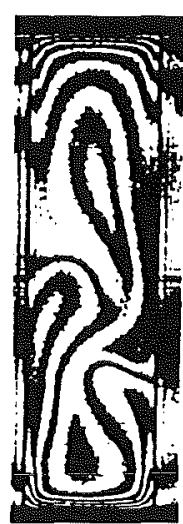

3

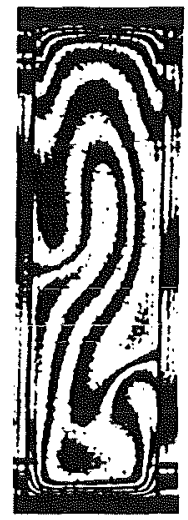

9

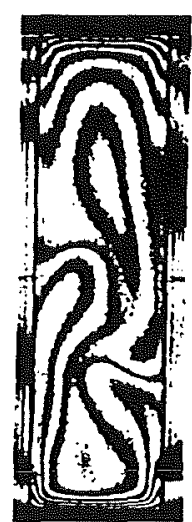

4

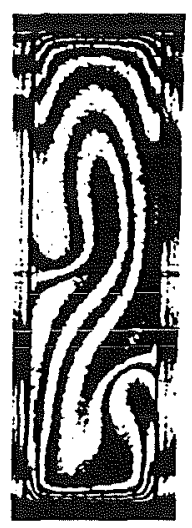

10

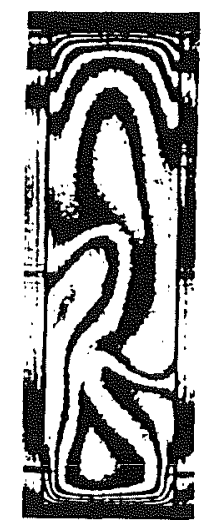

5

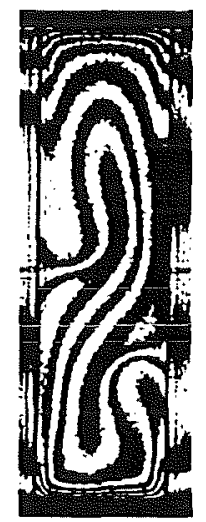

11

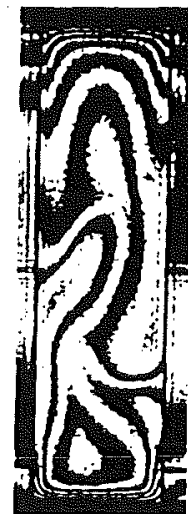

6

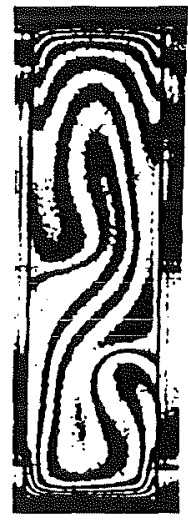

12

Fig. 2a - One period of a noisy oscillation at $\mathrm{Ra}^{*}=1.73$. The time interval between two interferograms is $\Delta t=5 \mathrm{~s}$. The oscillation period is $t \approx 70 \mathrm{~s}$.

Nonsymmetries in convective flow patterns are associated with so-called nonBoussinesq conditions. Such non-Boussinesq conditions most often arise from the variation of viscosity with temperature (Pa1m, 1960). Krishnamurti (1968) has shown that a nonlinear temperature profile in side walls produces effects similar to those produced by a variable viscosity. It appears from the fringe pattern in the side walls that the profile resembles that one investigated by Krishnamurti. From Krishnamurti's results it may be concluded that the nonlinearity of the temperature profile in the side walls of our Hele-Shaw slot produces the observed nonsymmetries of the flow patterns. 


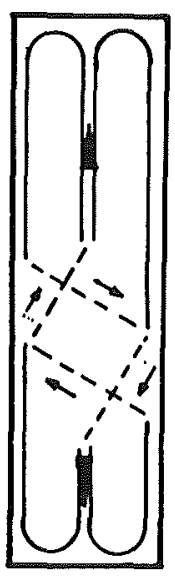

Fig. $2 b$ - Sketch of the flow pattern of the nonsymmetric two-sided oscillation. The flow pattern exhibits a cellular structure close to the horizontal boundaries and a central asymmetric exchange zone. The nonsymmetry results from two different exchange processes, one from one side to the centerline and one from side to side.

According to Curry, et a1. (1984) there exists an important link between symmetry and the picture of the transitions in oscillation character. In $I$, Koster \& Miller discussed the relation of the four roll mode picture with the time average structure of the oscillations. In the same way we define qualitative wave numbers for the time average flow pattern in this experiment. Applied to the visualized temperature fields, this model allowed the following observations: (i) Oscillations develop first in low wave number cells (lower boundary, figure $2 \mathrm{~b}$ ) and, at higher Rayleigh number, decay first in low wave number cells. This emphasizes individual stability behavior. (ii) Monoperiodic and frequency locked oscillations develop when instabilities in thermal boundary layers, monitored with local thermocouples, occur in a phase-locked sequence. Phase-locking of thermocouple signals develops when the plume movement in the central exchange zone is highly ordered. (iii) A subharmonic oscillation may be generated by periodically generated thermal plumes of periodically varying heat content, visualized by periodically varying fringe densities generated by the individual plumes. 
The two frequencies of highest power analyzed from the time dependent temperature signals at the five locations shown in figure 1 were, if incommensurate, transformed into dimensionless periods $\tau=x /\left(\mathrm{fh}^{2}\right)$ and plotted as a function of the normalized Rayleigh number $\mathrm{Ra}^{*}=\mathrm{Ra}_{\mathrm{R}} / \mathrm{Rasc}_{\mathrm{O}}$. These results are displayed in figure $3 a-e$. In order to satisfy the continuity in the $\tau$-Ra plots the frequencies will be indexed 1 or 2 . The sequences of the statistical phenomena observed in the range of time dependent flow is summerized by bar graphs in figure 4. We will discuss figures 3 and 4 now.

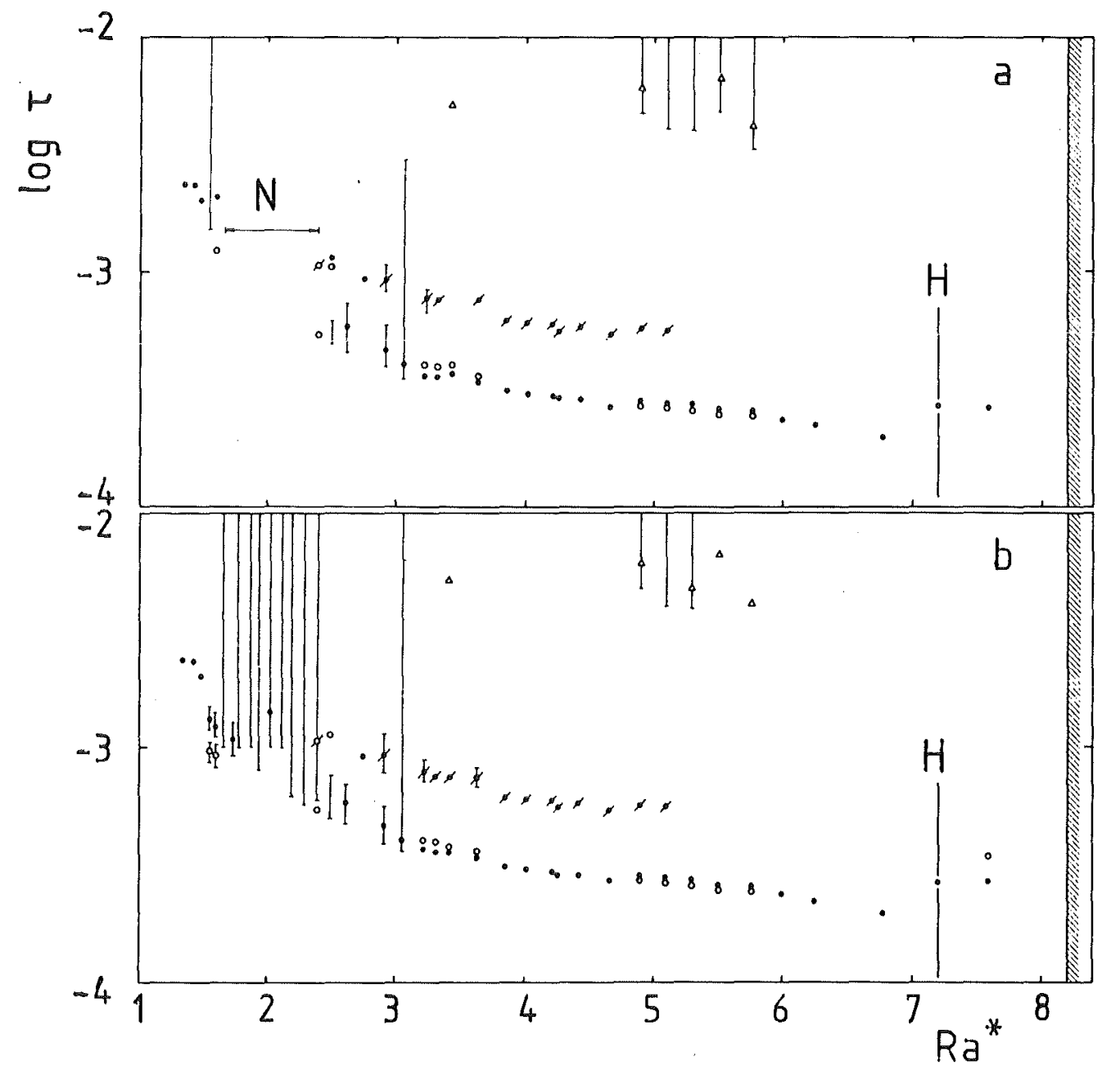

Fig. 3 - Dimensionless periods in the Hele-Shaw slot as a function of the normalized Rayleigh number $\mathrm{Ra}^{*}$. Diagrams a to e correspond to the transducers 1 to 5 . The symbol in the hysteresis bar is from the down-scan experiment. $\operatorname{Pr}=37.4 \pm 0.2, \mathrm{Ra}_{\text {osc }}=2.79 \times 10^{7}$. Symbols $\bullet, \mathrm{f}_{1} ; \mathrm{o}, \mathrm{f}_{2} ; \emptyset$ subharmonics $1 / 2 \mathrm{f}, \Delta$ beating period, (1) $\tau=1.93 \times 10^{-3}\left(\mathrm{Ra}^{*}\right)^{-2.3}$ 


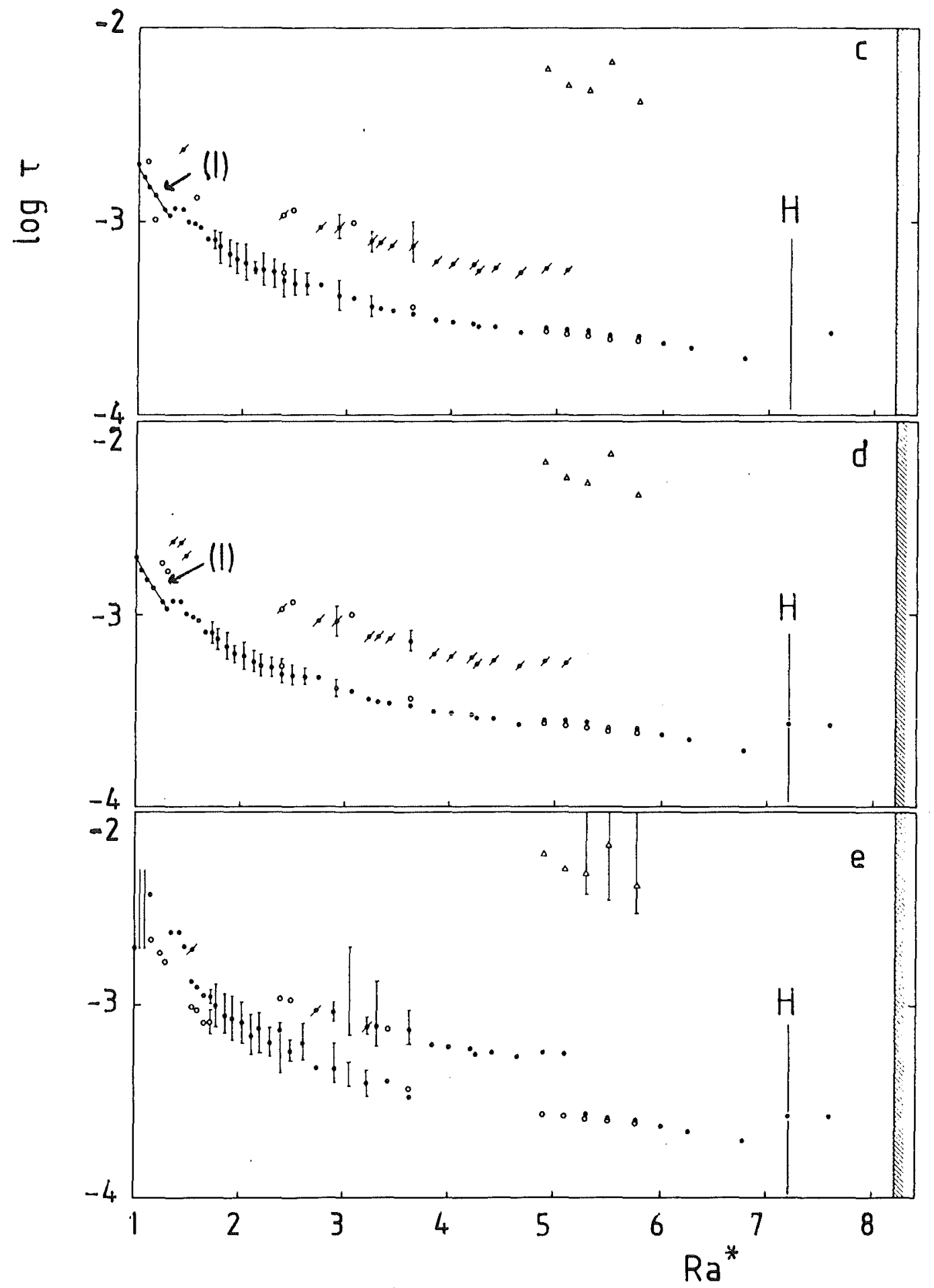




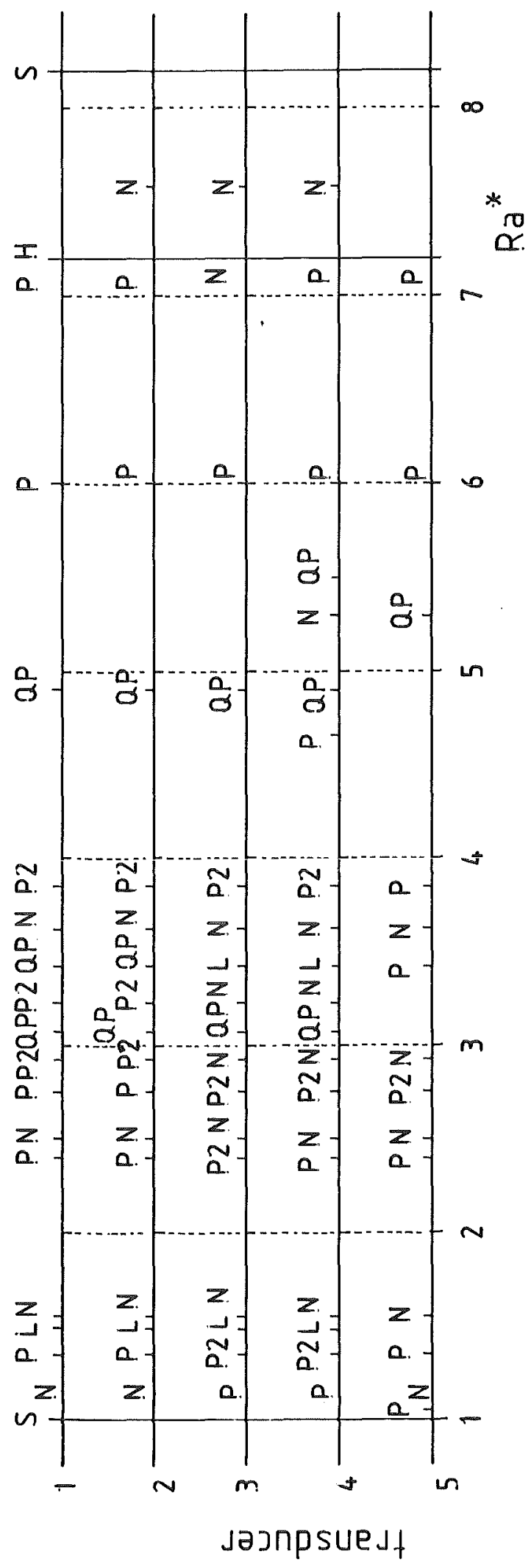

Fig. 4 - Bar graphs of the various oscillation states as a function of $\mathrm{Ra}^{*}$ and transducer. (S) steady; (P) periodic; (QP) quasiperiodic (biperiodic); (L) freqency-locking, (P2) subharmonic; (N) noisy, nonperiodic; (H) hysteresis. 
At first glance we see that the period decreases with increasing Rayleigh number, confirming the results in $I$. Generally, all five transducers monitor similar oscillation structures although the power of individual frequencies changes from location to location (see also Koster \& Müller, 1981).

A new finding is that time dependent signals are observed first at the two lower transducers 3 and 4 as a monoperiodic oscillation of frequency $\mathrm{mf}_{1}$, with $\mathrm{m}=1,2,3,4$. This means that the lower thermal boundary layers are now unstable. At this onset of oscillations at the lower boundaries, we define the critical Rayleigh number $\mathrm{Ra}_{\text {osc }}{ }^{\circ}$

At the upper two locations 1 and 2 , we detect only white noise up to $\mathrm{Ra}^{*}<$ 1.35. This means that the upper thermal boundary layers are stable in this range of Rayleigh numbers. Moore \& Weiss (1973) observed in their specific configuration that, at high Rayleigh numbers, thermal plumes may not convect within the whole fluid layer. They found that an element of fluid loses vorticity through friction. Our observation that plumes apparently dissipate before they reach the upper stagnation point may thus be caused by friction of the narrow gap flow.

At location 5 at midheight of the slot, a periodic oscillation is measured at $\mathrm{Ra}=\mathrm{Ra}$ osc . For $1<\mathrm{Ra}^{*}<1.35$, the signal at location 5 becomes quasiperiodic with high noise level and with a concurrent increase in period time similar to the findings in $I$. This reduction in signal to noise ratio (SNR) from the lower to the central thermocouple suggests also that the plumes, which are periodically breaking off the lower boundaries, dissipate to a certain extend when rising into the bulk fluid.

At the onset of oscillatory flow $\left(1 \leq \mathrm{Ra}^{*}<1.3\right)$, a correlation of the dimensionless period yields $\tau \sim \mathrm{Ra}^{-2.3}$ for the periodic oscillation close to the lower two thermal boundary layers, with a correlation coefficient $r=0.993$. The exponent is higher than the theoretical value of -2.0 required by the Howard model for Hele-Shaw cells (Koster \& Miiller, 1982). This disrepancy can not be explained at present. Another unidentified cause of instability must accelerate the oscillations.

At $\mathrm{Ra}^{*}=1.35$, a monoperiodic oscillation of signal to noise ratio $\mathrm{SNR}=50$ develops at the upper locations 1 and 2. The lower locations 3 and 4 then 
develop a subharmonic oscillation where the subharmonic frequency $\mathrm{f}_{1} / 2$ has the same value as the basic frequency of a monoperiodic oscillation at the upper locations 1 and 2. The oscillation character at location 5 is also monoperiodic at a freqeuncy which is the subharmonic at locations 3 and 4 . Both the lower and upper thermal boundary layers are now unstable.

With increasing $R a$ the signals become noisy after a weakly locked oscillation. At the location 1 and 2 , the noise character is of the low pass type whereas, at locations 3 and 4 , it is of the band pass type. Location 5 is influenced by both types of noise.

At $\mathrm{Ra}^{*}>2.3$, the noisy oscillations, for a certain length of time, often hours in duration, may change intermittently into a well ordered oscillation.

In the range $3.85 \leq \mathrm{Ra}^{*} \geq 4.66$, a subharmonic oscillation of high SNR (SNR > $10^{3}$ ) develops at all four corners. An important feature of this oscillation is, that again, at location 5, the oscillation is monoperiodic. The basic frequency of this periodic signal equals the value of the subharmonic at the horizontal boundaries. The temperature signals and the power spectra from all 5 locations of such on oscillation at $\mathrm{Ra}^{*}=4.01$ are displayed in figure 5 .

In the range $4.90 \leq \mathrm{Ra}^{*} \leq 5.77$, a biperiodic (two frequencies $\mathrm{f}_{1}$ and $\mathrm{f}_{2}$ ) flow of high SNR develops. The temperature signals exhibit beating oscillations. The power spectra analyzed from the temperature signals at $\mathrm{Ra}^{*}=4.90$ are displayed in figure 6.

At the beginning of the range of these beating oscillations, the subharmonic at the upper and lower transducers is the basic frequency with highest power at location 5. When the subharmonics disappear, the frequency $f_{1}$ of the upper locations 1 and 2 develop the highest power at location 5 . 


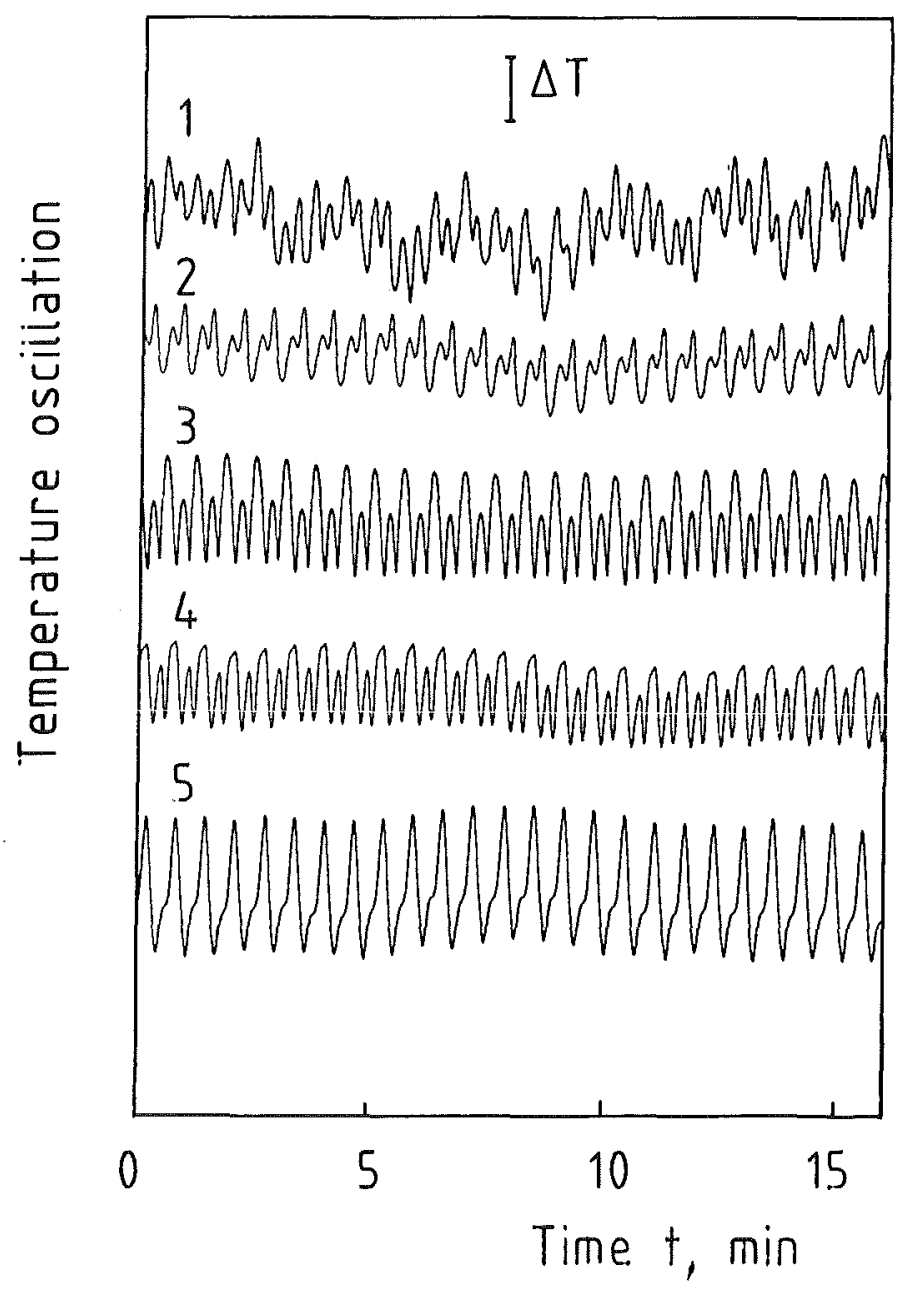

Fig. 5a - Temperature signals at $\mathrm{Ra}^{*}=4.01$ leading to the power spectra in figure $5 \mathrm{~b}$. The numbers indicate the location of the thermocouples in figure 1. The amplitude of the temperature oscillation $\Delta \mathrm{T}$ has following tabulated values at the transducers:

\begin{tabular}{l|ccccc} 
Transducer No. & 1 & 2 & 3 & 4 & 5 \\
\hline$\Delta \mathrm{T}(\mathrm{K})$ & 0.15 & 0.3 & 0.15 & 0.07 & 0.15
\end{tabular}




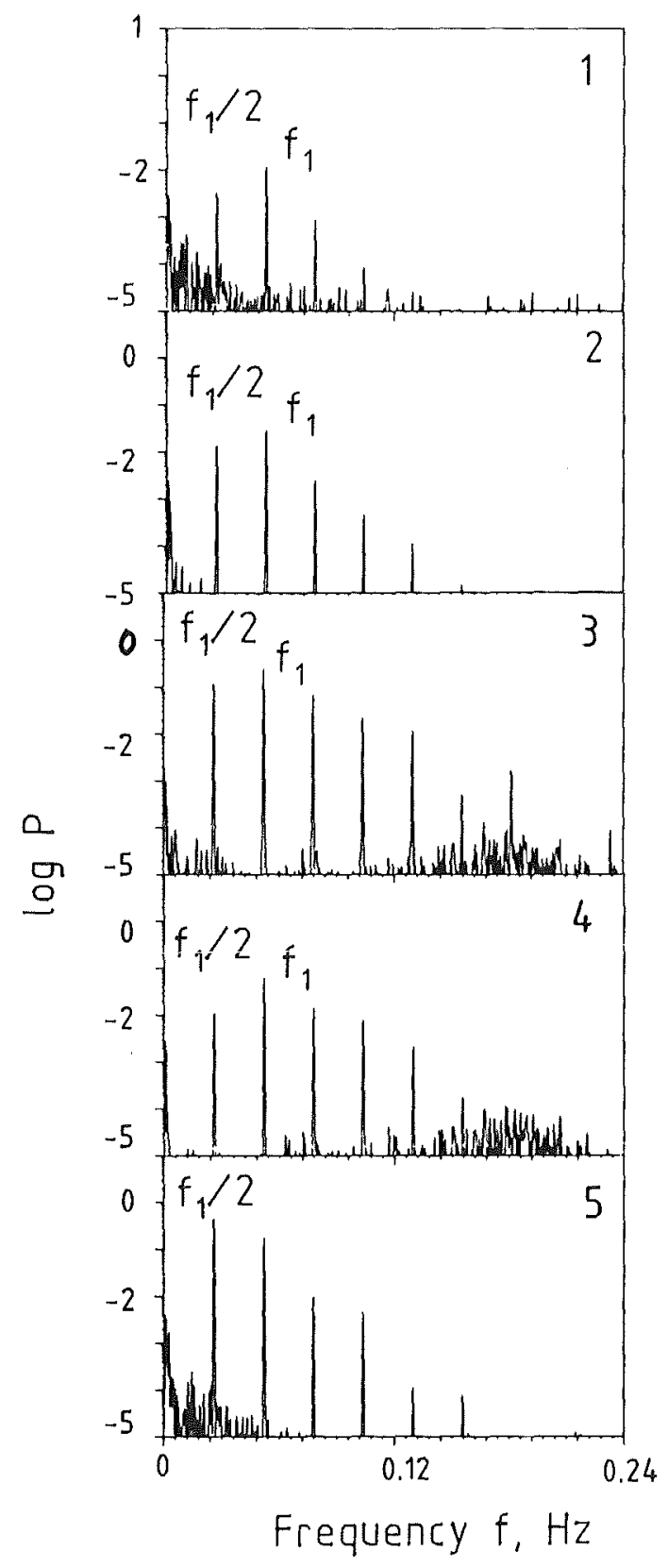

Fig. 5b - Power spectra of a complicated periodic oscillation at $\mathrm{Ra}^{*}=4.01$. At transducers 1 to 4 the oscillation has a subharmonic character whereas at transducer 5 the oscillation is monoperiodic. 


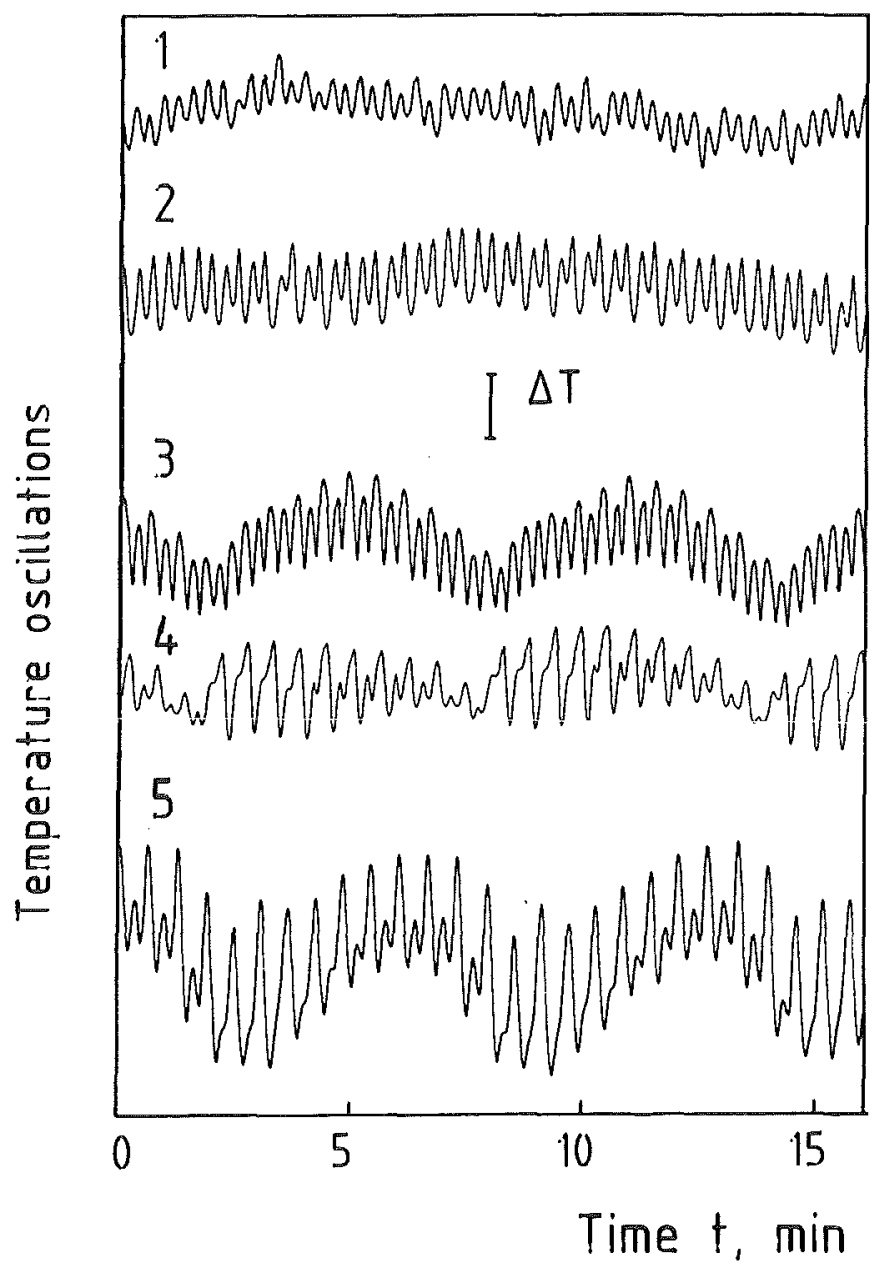

Fig. 6a - Temperature signals at $\mathrm{Ra}^{*}=4.90$ leading to the power spectra in figure $6 \mathrm{~b}$. The numbers indicate the location of the thermocouple in figure 1. The amplitude of the temperature oscillation $\Delta T$ has following tabulated values at the transducers:

\begin{tabular}{l|ccccc} 
Transducer No. & 1 & 2 & 3 & 4 & 5 \\
\hline$\Delta T(K)$ & 0.3 & 0.3 & 0.15 & 0.07 & 0.15
\end{tabular}




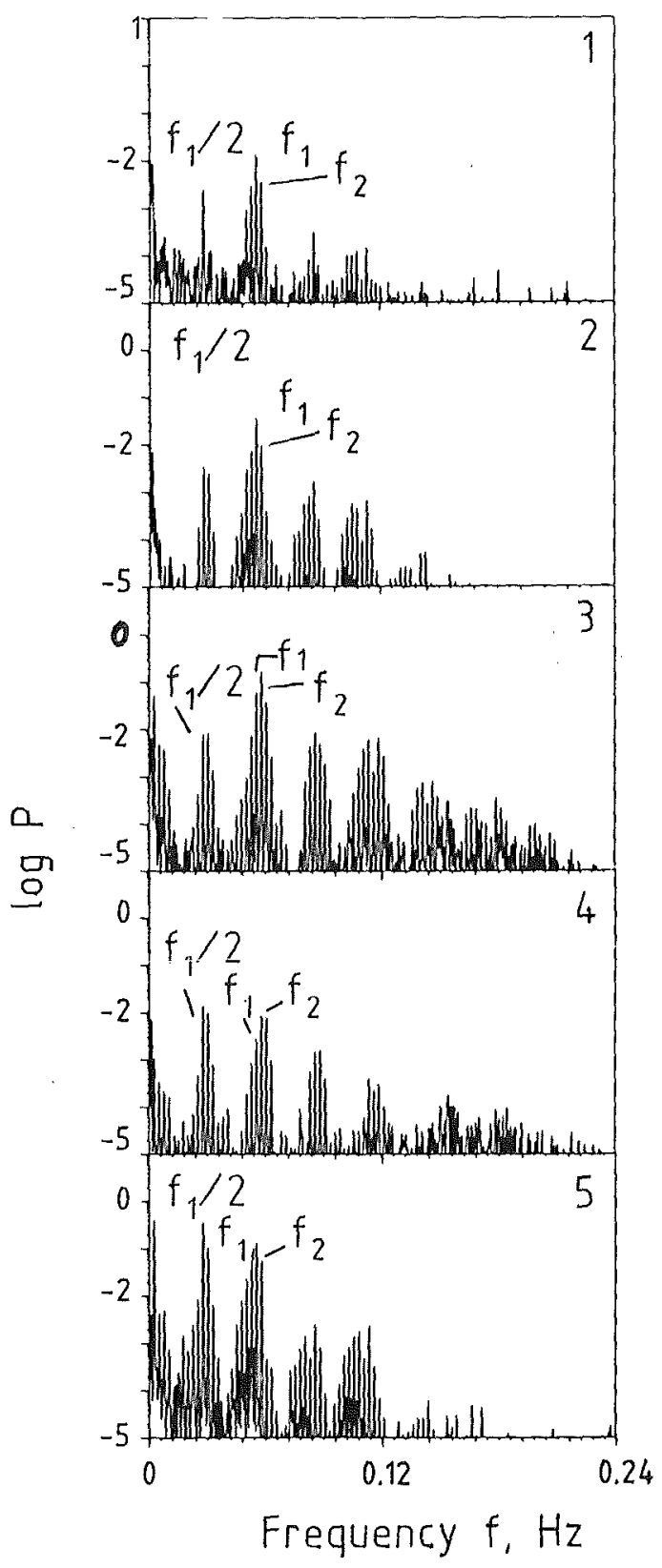

Fig. $6 \mathrm{~b}$ - Power spectra of a biperiodic oscillation at $\mathrm{Ra}^{*}=4.90$. At transducers 1 and 2 the frequency $f_{1}$ has the highest power and at transducers 3 and 4 the frequency $f_{2}$ has a higher power than $f_{1}$, but the frequency $\mathrm{f}_{1} / 2$ has the highest power at transducers 4 and 5 . 
The Fourier analysis shows that the two frequencies $f_{1}$ and $f_{2}$ are very close together and coupled nonlinearly, thus producing numerous sidebands of $\Delta=$ $\mathrm{f}_{2}-\mathrm{f}_{1}$. The important feature of these oscillations is that the frequencies $f_{1}$ and $f_{2}$ have different power at the different locations: $f_{1}$ has the highest power at locations 1 and 2, where high wave number roll cells are observed, and $\mathrm{f}_{2}\left(\mathrm{f}_{2}>\mathrm{f}_{1}\right)$ has the highest power at locations 3 and 4 , where low wave number roll cells are observed. This type of beating oscillation is observed only in nonsymmetric flow patterns.

This measurement is interpreted as follows: (i) One, or two phase-locked, instability event(s) occur at a defined frequency $f_{1}$ at the upper thermal boundary layers whereas a second (phase-locked) event occurs at a higher frequency $\mathrm{f}_{2}$ at the lower thermal boundary layers. (ii) The lower, low wave number roll cells experience a higher frequency of instability events than the upper high wave number roll cells. (iii) The asymmetry of the flow pattern allows phase coupling of the instability events occurring at different frequency.

At $\mathrm{Ra}^{*}=6.0$, the flow oscillations become monoperiodic again with the same basic frequency $f_{1}$ at all locations. Only the power and the number of harmonics differ. Instability events occur now at the frequency $f_{1}$ in all horizontal thermal boundary layers. With increasing Rayleigh number the power of the frequencies decreases. The low wave number rolls exhibit the weaker oscillations. The period of oscillation decreases at first with increasing Ra, but then increases again. Fina1ly, the amplitude at all locations drops to the white noise level. Judging from the power of the frequencies, it appears that the oscillations die of first at the lower boundaries where the low wave number rolls exist. We define as critical Rayleigh number ( $\mathrm{Ra}^{*}=8.20$ ) of the reverse to steady state four roll flow (c. f. I), the Rayleigh number at which the power $\mathrm{P}$ of the monoperiodic oscillations drops below $\mathrm{P}=10^{-5} \mathrm{~K}^{2} / \mathrm{Hz}$ (white noise level) at all 5 locations.

The flow remained steady until $\mathrm{Ra}^{*}=11.31$. When decreasing the temperature difference quasisteadily, a monoperiodic oscillation reappears at $\mathrm{Ra}^{*}=7.19$ at all locations but location 3 where noise persists. Now three thermal boundary layers are unstable. We might have missed individual onsets of oscillation in the thermal boundary layers by changing $\Delta^{T}$ by too large a decrement. Again, the power of the frequencies was quite different at each location. 
This observed hysteresis has a value of about $\Delta \mathrm{Ra}^{*}=1$ and is of the same quality as in $\mathrm{I}$.

\subsection{Correlation Analysis}

In this paragraph we will investigate the correlation of temperature signals as a function of delay times. We present result at specific Rayleigh numbers. First we will analyze the auto-correlation function, ACF, of all five temperature signals after the onset of oscillatory flow. Results are shown in figure 7.
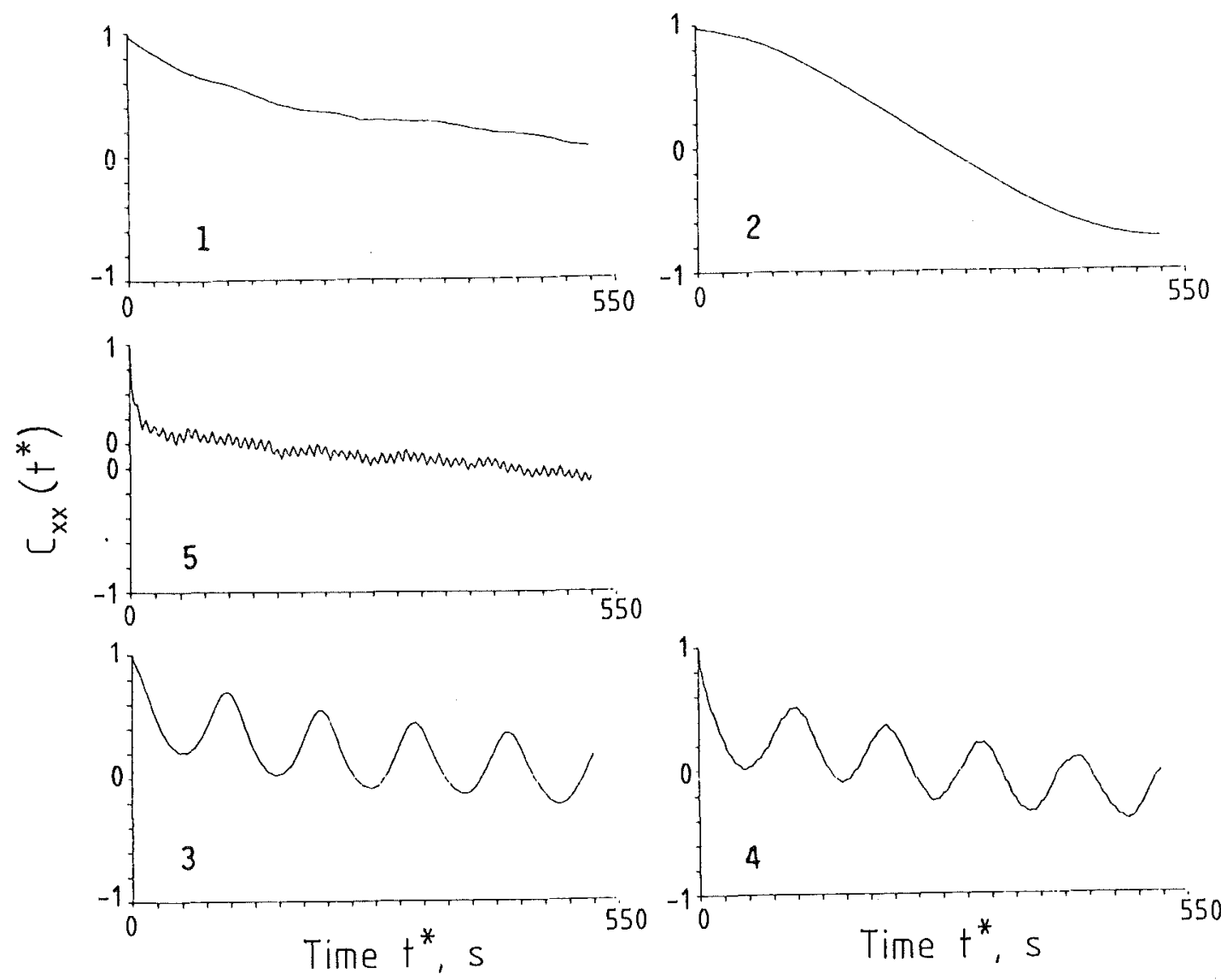

Fig. 7 - Auto-correlation functions of the temperature signals at the five locations at $\mathrm{Ra}^{*}=1.05$. 
As already indirectly derived from the analysis of the power spectra in section 3.2, the flow oscillations at $\mathrm{Ra}^{*}<1.35$ develop at the two lower thermal boundary layers. The transducers 3 and 4 at the lower horizontal boundaries, however, show the occurrence of periodic events in the temperature signals. The period $\Delta t^{*} \sim\left(\mathrm{f}_{1}\right)^{-1}$ give the time delay between the occurrence of two instability events. The ACF's prove that no periodic signal can be detected at the upper transducers 1 and 2, except for some slow movement. The periodic higher frequency ripple observed at transducer 5 suggests dissipation of the visualized thermal plumes that come from the lower thermal boundary layer areas.

The cross-correlation function, CCF, of the two lower periodic signals (figure 8)
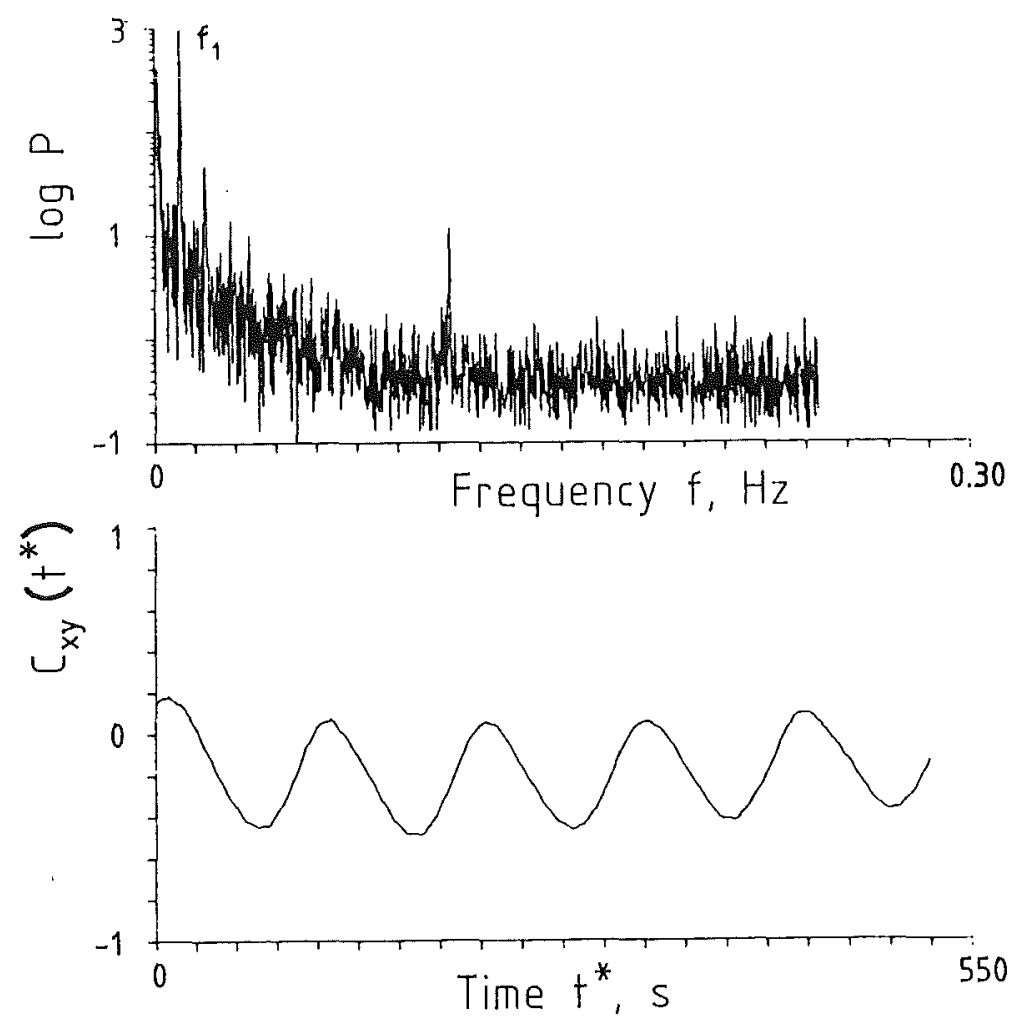

Fig. 8 - Cross-correlation function and cross spectrum of the lower signals 304 . 
show that, (1) the instability events in both thermal boundary layers are phase-locked, (ii) the delay time of the plumes, one passing the transducer on the lower left side and another passing the transducer on the right side is $\Delta t^{*}=9 \mathrm{sec}$. Compared to the oscillation period of $105 \mathrm{sec}$ and considering the separating central stagnation flow (section 3.1) and that the thermal plume moves away from both transducers, this means that two different individual (but phase-locked) physical instability events cause the oscillations in the lower thermal boundary layer areas.

Cross-correlation coefficients versus time delay of the temperature signals obtained at $\mathrm{Ra}^{*}=4.01$ (figure $5 \mathrm{a}$ ), at the five transducers are presented in figure 9. They show that the signals from all transducers, and thus the visualized plumes coming from the unstable thermal boundary layer areas, are phase-locked. The pattern of the correlation is periodic in multiples of the subharmonic period $2 \Delta t^{*} \sim 2 / f_{1}$ with almost constant amplitude of correlation. The oscillation is better correlated in delay times of the subharmonic period $2 \Delta t^{*} \sim 2 / f_{1}$ than the harmonic period $\Delta t^{*} \sim 1 / f_{1}$.

More specifically, the CCF of the temperature signals at the lower transducers 3 and 4 shows that: (i) The coupling of the two instability events at the lower thermal boundary layers is strong with $\left(\mathrm{C}_{\mathrm{xy}}\left(t^{*}\right)\right)_{\max }=+0.98$. (ii) The two signals are shifted in time, as the first maximum is delayed by $\Delta^{*}=3 \mathrm{sec}$. This means that the thermal instability event occurs earlier in the area of the thermal boundary layer close to transducer 3. Again, the delay time is much smaller than the period time of the oscillation, giving evidence that, at the lower boundary, two individual instability events drive the flow oscillation. The CCF of the signals at the upper transducers 1 and 2 reveals: (i) That these signals are less strongly correlated with $\left(C_{x y}\right)_{\max }=$ 0.68 . 

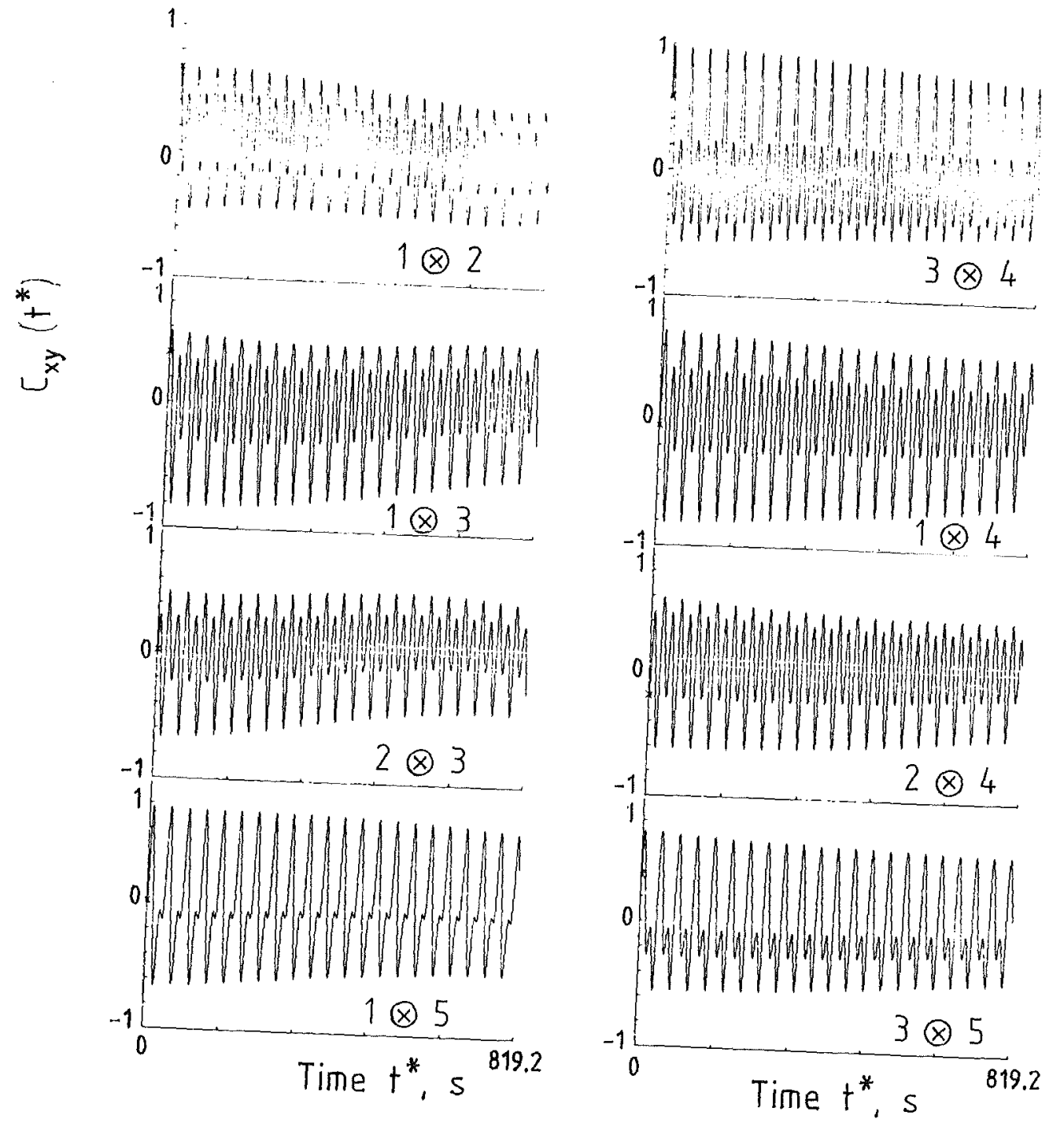

\footnotetext{
Fig. 9 - Cross-correlation functions of the subharmonic oscillation at $\mathrm{Ra}^{*}=$ 4.01. Transducer numbers are marked; the second one includes the time displacement.
} 
(ii) The thermal instability events are synchronous, since the maximum of $C_{x y}$ ist at delay time $\Delta t^{*}=0 \mathrm{sec}$. This means that the instability events in the upper two thermal boundary layers occur simultaneously. Consideration of the rising central stagnation flow gives evidence that two synchronous individual instability events drive the flow oscillation from above.

The signals from the transducers mounted at the same vertical side wall, 1 and 3,2 and 4 , respectively, are correlated weakly with $\left(C_{x y}\right)_{\max }=0.60$ and each set being phase shifted somewhat differently. Those from the transducers mounted in one diagonal, 1 and 4 , are correlated with $\left(C_{x y}\right)_{\max }=0.75$. The second diagonal, between transducers 2 and 3 , has lower correlation, $\left(C_{x y}\right)_{\max }$ $=0.50$, than the first diagonal, which is expected since the flow pattern (section 3.1) is asymmetric. Again, the instability events are phase shifted.

Cross-correlating the temperature signals from the horizontal thermal boundary layer areas with those measured at mid-height of the slot emphasizes a strong correlation at the subharmonic frequency $\mathrm{f}_{1} / 2$. The events occurring at the frequency $f_{1}$ have low correlation. This is expected since the visualization shows that thermal plumes from both lower thermal boundary layer areas, phase-shifted by $\pi / 2$, alternatively pass by transducer 5 at the frequency of $\mathrm{f}_{1}$, which increases the power of $\mathrm{f}_{1} / 2$.

Cross-correlograms of the quasiperiodic oscillation observed at $\mathrm{Ra}^{*}=4.90$ (cf. figure 6) are shown in figure 10. The degree of correlation of the frequencies in this flow is generally lower than at $\mathrm{Ra}^{*}=4.01$, but varies more strongly with delay time. Low correlation as well as maximum correlation appears in multiples of the period $\Delta t^{*} \sim 1 /\left(f_{1}-f_{2}\right)$ where $f_{1}$ and $f_{2}$ are the two frequencies analyzed from the spectra (figure $6 \mathrm{~b}$ ) calculated for the upper and lower areas respectively. In a manner analogous to the observations at $\mathrm{Ra}^{*}=4.01$, the plumes develop in phase, $\emptyset=0$, at the upper boundaries, and, with phase $\emptyset>0$, at the lower boundaries, which classifies them as four different instability events. As seen also from these spectra, the CCF shows that oscillations in the lower thermal boundary layer areas exhibit a stronger subharmonic content than their upper counterparts. Nonperiodic flow occurs when the two lower and upper horizontal adjacent signals are no longer correlated. This means that at least three or four incommensurate instability events, in different locations, cause the transition to turbulent flow. Turbulent thermal flow causes a stochastic refractive index in the central ex- 
change zone, which is also visualized as a chaotic fringe movement in the interferograms.
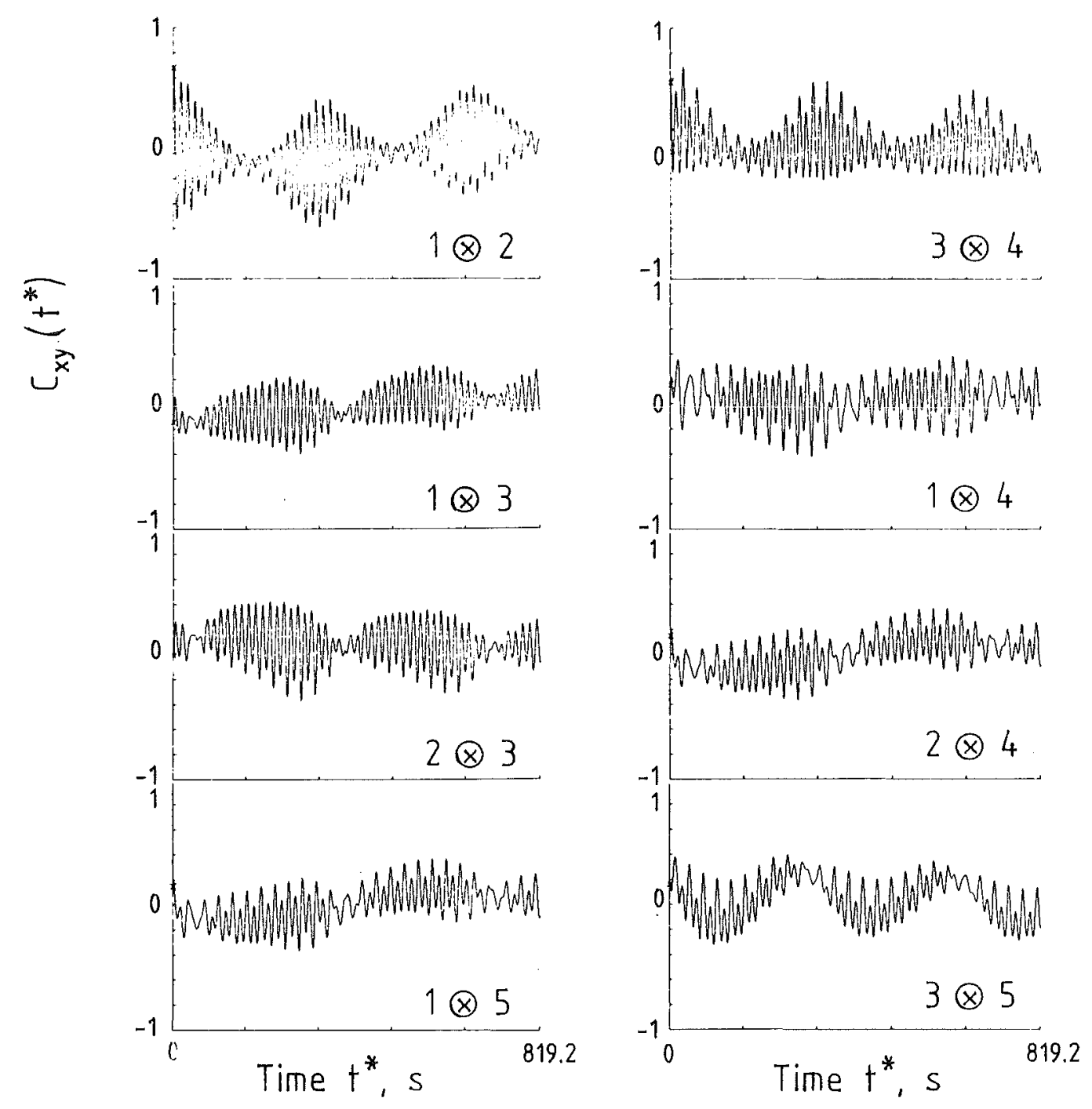

Fig. 10 - Cross-correlation functions of other biperiodic oscillation at $\mathrm{Ra}^{*}=4.90$. 


\section{Discussion}

Local temperature measurements indicate that the stability of the horizontal thermal boundary layers play a major role in forming the statistical character of the flow oscillations. This, however, is not sufficient to demonstrate that the actual cause of the oscillatory character of the flow is intrinsic to the thermal bundary layers as no secondary vortices have been visualized in horizontal thermal boundary layer areas. The investigation, as set up, could not clarify whether the vertical bundaries and corners contribute any instability cause that affects time dependent flow, and especially the stability of the thermal boundary layers. It seems likely that thermal bridging, especially in the corner sections and the stagnation point type flow along the vertical side walls contribute to the unsteady character of the flow. This problem is subject to further study (Zimmermann, et al., 1986). Nevertheless, Koster \& Müller (1982) indentified the stagnation point as the area where the temperature gradient is steepest and where secondary vortices deve1op. Second, the measuring thermocouples are located downstream of the stagnation points. Therefore, for purpose of this experiment, we discuss instability events as events that occur at the locations where the thermocouple measurements were taken namely in the horizontal thermal boundary layers.

As a result of the flow pattern analysis, the character of the flow oscillations depends on the flow pattern, which also varies with the Rayleigh number. This result substantiates findings in $\mathrm{I}$ and results by McLaughlin \& Orszag (1982) as well as Curry, et al. (1984) who recognized the important link between the symmetry breaking modes and the oneset of turbulence. Roughly, we observed the following evolution of oscillations with increasing $\mathrm{Ra}^{*}$ (figure 4): Steady, monoperiodic, complicated periodic (biperiodic), nonperiodic, subharmonic, biperiodic, monoperiodic, steady. Curry, et a1. (1984) found that only two dimensional flow can revert from quasiperiodic to monoperiodic oscillations with increasing Rayleigh number. With that result it appears that the flow in the Hele-Shaw slots, usually considered two dimensional (Bear, 1972), changes dimensionality: from a 2D-flow at low Rayleigh numbers to a $3 \mathrm{D}$-flow in the range of complicated time dependent flow, back to a $2 \mathrm{D}$-flow at very high Rayleigh number. In the present study, no evaluation of flow dimensionality was done because (i) the Hele-Shaw slot is a narrow gap and visualization of the area $h \mathrm{x} d$ was not possible and (ii) interferometry is an integrating technique along the light path. 
In the present study, the mechanism of the oscillations was always monitored close to the horizontal thermal boundary layers. Major results of the osci1latory flow analysis are:

(i) In the range $1 \leq \mathrm{Ra}^{*}<1.35$ oscillations develop solely in the lower thermal boundary layer areas. (ii) In a biperiodic regime, two frequencies may develop, the first having the highest power and orginating in another thermal boundary layer than the second. (iii) Subharmonic oscillations are observed in the horizontal thermal boundary layer areas but not at midheight of the fluid layer. (iv) Subharmonic oscillations at the bundaries may occur concurrently with a monoperiodic oscillation at some point located at midheight of the cavity. These results indicate the individual character of the individual instability events.

Major results of the correlation analysis are: (i) Individual instability events occur in the four horizontal thermal boundary layers. (ii) The amplitude of correlation of monoperiodic, subharmonic and biperiodic flow is periodicaliy dependent on the delay time. (iii) Unsteady events in horizontally adjacent thermal boundary layers couple more strongly than those in more distant lower and upper ones. (iv) Instability events which are phase-shifted by $\emptyset \geq 0$ may be phase-locked, resulting in a periodic oscillation of commensurate frequencies. ( $v$ ) Turbulent flow occurs when the signals from the vertically and horizontally adjacent transducers are no longer correlated. These results prove that several instability events develop independently.

The amount of cross- correlation of two and more interacting oscillators is determining the statistical character of the flow oscillations (c. f. Grebogi, et al., 1985). A periodic oscillation with one frequency $f_{1}$ is expected if there is only one time dependent instability event in the fluid layer or if more events in one fluid layer are absolutely uncoupled (Koster, et al., 1986). Periodic flow also develops if several phase-locked events of frequency $f_{1}$ are strongly correlated. More complicated periodic oscillations which encompass two incommensurate frequencies $f_{1}$ and $f_{2}$ show a variable amount of correlation over a period $\left(f_{1}-f_{2}\right)^{-1}$. Turbulent flow develops if there is no correlation between different instability events.

The Newhouse, Ruelle \& Takens (1978) model, (i) says that nonperiodicity may develop if a third incommensurate oscillator develops in the fluid layer, 
and, (ii) specifies the sequences of transitions from steady, to biperiodic, to nonperiodic. The Hele-Shaw slot convection flow, however, exhibits more than three bifurcations, including appearance and disappearance of certain types of oscillations and a reverse transition to steady flow. Therefore, the Newhouse, Ruelle \& Takens model describes the flow behavior in our specitic experiment only in terms of the maximum number of independent oscillatory modes which a system can sustain without generating broadband frequency spectra.

According to the present and previous (Koster, et al., 1986) cross-correlation results, the findings of Grebogi, et al. (1983, 1985) saying that the statistical behavior of a many oscillator system is a function of a nonlinearity parameter between individual oscillators are most important. 


\section{Summary and Conclusion}

Convection experiments were performed in a Hele-Shaw slot with low conductivity thermal boundary conditions. The slot was filled with silicone oil of Prandt 1 number $\mathrm{Pr} \cong 37$ and was heated from below and cooled from above. Thermocouples protruded into the liquid layer at four corners of the slot in order to monitor thermal perturbations in the four horizontal thermal boundary layers.

The major results of the present study are: (i) Unstable horizontal thermal boundary layers are a cause of time dependent convection. It could not be clearified unambiguously whether the cause of the oscillations is always and exclusively based on the Howard principle of the unstable thermal boundary layer. (ii) If there is one instability event in any horizontal thermal boundary layer, this event will be a contributing driving mechanism to the oscillation. (iii) The criterium for instability must be met separately by each individual thermal boundary layer. The Koster \& Miiller (1984) conjecture on independence of the events is shown to be corredt. (iv) Different instability events in one fluid layer may develop different frequencies of instability. (v) The amount of correlation of three and more interacting instability events has an effect on whether the oscillation is ordered or chaotic. (vi) Fluid dynamic mechanisms of subharmonic and locked ocillations have been identified as phase-coupled thermal events that appear to develop in different areas. A subharmonic oscillation may be caused by phase-locked thermal instability events of periodically varying intensity.

A major conclusion drawn from the experiments in this paper, confirming Grebogi et al. (1983), is the following: The richness in dynamical behavior of time dependent convection in high Prandtl number fluids confined in small boxes is produced by a variable amount of nonlinear coupling of individually behaving instability events monitored in thermal boundary layer areas of adjacent roll cells.

The author wishes to express his gratitude to U. Miller for his continued interest in this study. 


\section{References}

Ahlers, G., 1974 Phys. Rev. Lett. 33, 1185.

Ahlers, G. \& Behringer, R. P., 1978a Phys. Rev• Lett. 40,712.

Ahlers, G. \& Behringer, R. P., 1978b Progr. Theor. Phys - Supp1. 64,186.

Bear, J., 1972 Dynamics of Fluids in Porous Media, Elsevier.

Bendat, J. S. \& Piersol, A. G., 1971 Random Data, New York: Wiley.

Berge, P.\& Dubois, M., 1976 Optics Commun. 19, 129.

Berge, P.\& Dubois, M., 1979 J. Physique Lett. 40, L505.

Busse, F. H. \& Whitehead, J. A., 1974 J. Fluid Mech. 66, 67 .

Curry, J. H., Herring, J. R., Loncaric, J. \& Orszag, S. A., 1984 J. Fluid

Mech. 147,1 .

Gollub, J. P. \& Benson, S. V., 1978 Phys. Rev. Lett. $41,948$.

Gollub, J. P. \& Benson, S. V., 1980 J. Fluid Mech. 100, 449.

Gray, D. D. \& Giorgini, A., 1976 Int1. J. Heat Mass Transfer 19, 545.

Grebogi, C., Ott, E. \& Yorke, J. A•, 1983 Phys• Rev• Lett• 51, 339.

Grebogi, C., Ott, E.\& Yorke, J. A., 1985 Physica 15D, 354.

Hopf, E., 1948 Comm. App1. Maths. 1, 303.

Howard, L. N., 1964 in: Proc. 11 th Int. Congr. Appl. Mech., München, (ed. H.

Görtler), p. 1109, Springer.

Koster, J. N., 1983 Experiments in F1uids 1, 121.

Koster, J. N., 1985 Int. Comm. Heat Mass Transfer, 12, 1959.

Koster, J. N. \& Muiller, U., 1981 Phys. Rev. Lett. 47, 1599.

Koster, J. N. \& Müller, U., 1982 J. Fluid Mech. 125, 429.

Koster, J. N. \& Müller, U., 1984 J. F1uid Mech. 139, 363.

Koster, J. N., Ehrhard, P.\& Müller, U., 1986 Phys. Rev• Lett. 56, 1802.

Krishnamurti, R., 1968 J. Fluid Mech. 33, 445.

Landau, L. D. \& Lifshitz, E. M•, 1959 Fluid Mechanics, Addison-Wesley•

Libchaber, A. \& Maurer, J., 1978 J. Physique Lett. 39, L369.

Libchaber, A. \& Maurer, J., 1980 J. Physique Co11oq. 41, C3-51.

Malkus, W. V. R., 1954a Proc. Roy. Soc. A225, 185.

Malkus, W. V. R., 1954 b Proc. Roy. Soc. A225, 196.

McLaughlin, J. B. \& Orszag, S. A., 1982 J. Fluid Mech. 122, 123.

Mitche11, W. T. \& Quinn, J. A., 1966 AIChE J1. 12, 1116.

Moore, D. R. \& Weiss, N. O., 1973 J. F1uid Mech. 58, 289.

Newhouse, S., Rue11e, D. \& Takens, F., 1978 Commun. Math. Phys. 64, 35.

Palm, E. 1960 J. Fluid Mech. 8, 183.

Rue1le, D.\& Takens, F., 1971 Commun. Math. Phys. 20, 167. 
Somerscales, E. E. C. \& Dropkin, D., 1966 Int1. J. Heat Mass Transfer 9, 1189 .

Sparrow, E. M., Husar, R. B. \& Goldstein, R. J. 1970 J. Fluid Mech. 41, 793. Walden, R. W., Kolodner, P., Passner, A. \& Surko, C. M., 1984 Phys. Rev. Lett., 53, 242 .

Willis, G. E. \& Deardorff, J. W., 1965 Phys. Fluids 8, 2225.

Willis, G. E. \& Deardorff, J. W., 1970 J. Fluid Mech. 44, 661.

Zimmermann, G., Ehrhard, P. \& Müller, U., 1986 personal communication. 